\title{
A Study of Japanese and English Versions of Stroop and Reverse-Stroop Tests in Japanese Elementary School Higher Grade Students' English Activities
}

\author{
Yasuyuki SAKUMA \\ Fukushima University
}

\begin{abstract}
Paying attention to language input is important for the memorization of language (Robinson, 2003). This study took high graders ( $5^{\text {th }}$ - and $6^{\text {th }}$ - grade students) engaged in English class activities in 2007 to examine relation between English language abilities and two kinds of attention abilities. Reverse-Stroop and Stroop tests of two languages (the first language, Japanese (L1), and English as a Foreign Language (EFL)) were used in order to measure attention abilities (processing abilities) and selective attention abilities (automaticity) in access and storage of two languages. Jidoeiken, Junior STEP Bronze Test (Bronze) was used to measure English proficiency. The following four points form the main results: (1) Processing abilities in Ll were more predominant than those in EFL, and the upper group of English proficiency has higher processing abilities in both languages. (2) Though automaticity did not develop so much in the processing, not only of L1 but also of EFL, abilities of automaticity in $\mathrm{Ll}$ processing might be more predominant than those of EFL processing, irrespective of English proficiency. (3) There were different processing abilities, accessing verbal code and generating imagery code from long-term memory (LTM) between Ll and EFL, while, there were similar processing abilities, accessing imagery code and generating verbal code from LTM between L1 and EFL. (4) The upper group might have had greater processing abilities to attend to smaller units of information (as words and phrases). On the other hand, the attention abilities of the lower group in task switching might be poor in more complicated English language information. Overall, in this research, it was suggested that there were different kinds of language processing depending on the kinds of attention and languages ( $\mathrm{Ll}$ and EFL), and English proficiency.
\end{abstract}

\section{Introduction}

What is the scientific evidence for the effects "foreign language activities" have on language abilities? Though the Ministry of Education, Culture, Sports, Science and Technology introduces several qualitative and quantitative evaluations as evidence, it 
depends on teachers who are in charge of foreign language activities, and they are very confused about the selection of an appropriate evaluation (Sakuma, 2010). As for prior studies on foreign language activities, there has been much research on affective factors of English activities ${ }^{1}$ and but less on the effects of English proficiency on Junior high school (JHS) students. Besides, there has been little research on the relation between English performance and affective factors; some research one-shot data (Butler-Goto \& Takeuchi, 2006a, 2006b) and a longitudinal study in a private school (Takada, 2008) and a public school (Sakuma, 2009), but hardly any research on quantitative data of foreign language activities from a cognitive point of view.

The Ministry of Education, Culture, Sports, Science and Technology introduces overall objectives for the activities in the new course of study as follows: to form the foundation of pupils' communication abilities through foreign languages while developing the understanding of languages and cultures through a variety of experience, fostering a positive attitude toward communication, and familiarizing pupils with the sounds and basic expressions of foreign languages.

The characteristics of the objectives focus on communication abilities, positive attitudes and familiarizing pupils with the sounds and basic expressions of foreign languages. Needless to say, the activities are not a subject and there is no need to evaluate language knowledge through conscious learning, such as a subject. The activities are, so to speak, a kind of unconscious learning which is centered on basic daily vocabulary. The influences that foreign language activities have on English language acquisition can be considered to be so small. Therefore, in evaluating any small changes in the basic vocabulary, I think it important to collect micro levels of data from the cognitive research design. As for appropriate vocabulary, basic words which would occur frequently and be acquired unconsciously in daily school life are considered to be more appropriate.

In this paper, I will use five color words (blue, red, yellow, green and black), which elementary school higher grade children have already learned and have many opportunities to read and listen to, and administer two language version (L1 and EFL) of Stroop and reverse-Stroop effects which are regarded as an index for language acquisition process.

\section{Stroop and Reverse-Stroop Tests}

A Stroop test is employed to examine how we access and store lexical information. Stroop effect is regarded an effective index of automaticity ${ }^{2}$ in language acquisition processing (Posner \& Snyder, 1975). If language processing is considerably automatic, this effect occurs not only in $\mathrm{L} 1$ but also in the second language $\left(\mathrm{L}^{3}\right)$. In a Stroop test, when we see a color word, such as red, in an incongruent ink color, such as blue, we must ignore the word and name the ink color (i.e., say "blue" ). We usually find it 
very hard to respond this task, compared to a task to name the ink color of color patches. The different response times between naming the ink colors of incongruent color words and naming the ink color of color patches is called Stroop interference (Stroop, 1935), while the interference in ignoring the ink colors of the words and naming the words is reverse-Stroop interference. This does not occur in oral response. However, this interference occurs in a matching method that selects the color patch corresponding to the meaning of the word. The response times to select the color patches corresponding to the meaning of words in an incongruent color ink are longer than those in black ink (Flowers, 1975; Pritchatt, 1968). This is known as reverse-Stroop interference. As for prior studies in oral response testing of L1 and L2, there has been a lot of research on only Stroop interference, most of the participants being adults.

Concerning L2, most research has focused on bilingual lexical representations under the two conditions of within-language and between-language (Mägiste, 1984; Chen \& Ho, 1986; Osaka, 1990; Ikeda, Matsumi, \& Mori, 1994; Brauer, 1998). The condition of within-language is the combination of the same language between stimulus and response, such as $\mathrm{L} 1$ stimulus and $\mathrm{Ll}$ response, and the condition of between-language is that of different languages, such as L1 stimulus and L2 response, or the reverse.

In this paper, I will use "The Original Japanese Version of Stroop and Reverse-Stroop Test II" (Hakoda \& Watanabe, 2005) and "The English Version of Stroop and Reverse-Stroop Test II" (Hakoda, Watanabe, \& Matsumoto, 2007) which have been developed for group testing of the matching method and can be measured by both Stroop and reverse-Stroop interferences. Stimulus languages and response languages in both versions of tests are monolingual combination, under the condition of within-language. The contents of the tasks in both tests are completely the same, except for the languages (Japanese or English). As for prior studies in matching response that $\mathrm{I}$ administer in this paper, there are also only adults for participants. Using the Japanese version of this test and the English achievement test for students of several universities, Hakoda, Hirai, Shina, and Yanai (2002) reported significant positive correlation between ability to pay attention to language information and English proficiency.

The interferences of the Stroop and reverse-Stroop test administered in this research are shown in Figure 1, based on Hakoda and Sasaki (1991). These models explain reverse-Stroop interference and Stroop interference.

Reverse-Stroop Interference: Two primary codings (imagery coding of "green", verbal coding of "red") occur. Secondary coding, which generates imagery code (color code) from verbal code, occurs. The secondary coding is interfered with by green imagery coding in the primary coding. Tasks 1 and 2 in the reverse-Stroop test show attention abilities and are focused on attention to written words. Reverse-Stroop 
interference rate between tasks 1 and 2 shows selective attention ability.

Stroop Interference: Two primary codings which are the same as in reverse-Stroop interference occur. Secondary coding, which generates verbal code from imagery code (color code), is interfered with by verbal coding in the primary coding. Tasks 3 and 4 in the Stroop test show attention abilities and are focused on attention to colors. Stroop interference rate between tasks 3 and 4 also shows selective attention ability.

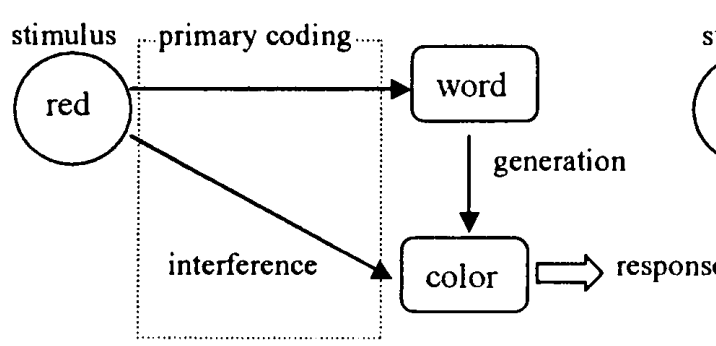

Reverse-Stroop Interference

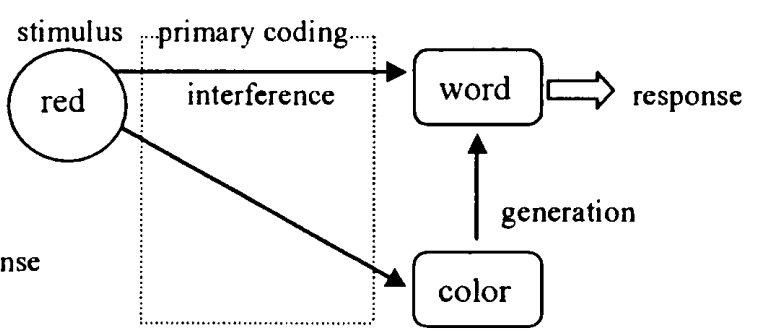

Stroop Interference

Figure 1 Models of Reverse-Stroop and Stroop Interferences, by Hakoda and Sasaki (1991).

Notes. The English translation of this model was done by the present author. The color naming (e.g. "red") in the Japanese version is written in hiragana characters (e.g. “あか”), not Chinese ones. Although written words of "red" in both interferences are shown in "green" ink, both verbal codings are "red."

In the present study, I focused on the characteristics of both attention abilities and selective attention abilities (interferences) between Japanese (L1) and English (FL) as well as between upper English proficiency and lower English proficiency in elementary school students. This research was based on the "Elementary School English Activities Promotion Project" in a mountain town for three years (school years 2005 to 2007). The present study was conducted to address the following five research questions concerning whether:

1. Scores of attention abilities (tasks 1 to 4 ) in the Japanese version of Stroop and reverse-Stroop tests are higher than those of the English version.

2. Interferences in the Japanese version of Stroop and reverse-Stroop tests are higher than those of the English version.

3. Scores of attention abilities (tasks 1 to 4 ) in English version of Stroop test in the higher level of English proficiency are higher than those in the lower level.

4. Interferences at the higher level of English proficiency are higher than those at the lower level.

5. There are some differences between the higher level of English proficiency and the lower level concerning the relation between the Japanese and English versions of Stroop and reverse-Stroop tests. 


\section{Method}

\subsection{Participants}

All 68 participants took part in English activities at an elementary school, in the form of team teaching (TT) with a homeroom teacher and an assistant language teacher (ALT). Most students depend on only school education, with few opportunities to learn English outside school, and they also had no experience of studying abroad. They had been studying English through 35 English activity classes ( 45 minutes per class) a year for three years since the 2005 school year. They had already learned Roman letters and thus were familiar with the English alphabet through English activities and various notices in the school. They could at least read words of five colors (blue, red, yellow, green and black) and had many opportunities to recognize these five words in their daily school life. Those participants considered unsuited to taking Stroop and reverse-Stroop tests as a result of misunderstandings, careless mistakes, lack of seriousness or failure to recognize five colors in the English alphabet, were removed in advance from the list of participants in this study.

\subsection{Materials}

The Bronze ${ }^{4}$ was the 2 nd Bronze test in 2007. It was administered as an index of English language proficiency. This test is for listening comprehension abilities of elementary school students with multiple choices. It is composed of three main sections, such as (1) words and phrases, (2) conversations and (3) sentences.

"The Original Japanese Version of Stroop and Reverse-Stroop Test II" and "The English Version of Stroop and Reverse-Stroop Test II" were used to examine attention abilities and selective attention abilities of $\mathrm{L} 1$ and EFL. The two versions of the tests have completely the same contents, except for the languages (Japanese or English version), and each test is composed of four kinds of tasks. Each task has 100 items. Each task takes 60 seconds to administer. Before the performance of each authentic version of a task, each practice version of a task was performed for several minutes, including questions and answers between students and a homeroom teacher. Then, a short break for about 30 seconds was given after each authentic version of the task. Thus, each test progresses as follows: practice 1, task 1, a break; practice 2, task 2, a break; practice 3 , task3, a break; practice 4 , and task 4 . There was a break for several minutes after the first version of the test. In addition, the order of administration of the two language versions of the tests was counterbalanced.

\subsection{Procedures}

The Bronze and two (Japanese and English) versions of tests were administered in each classroom. All the students took the 2 nd Bronze test together through the school public-address system at the end of October in 2007. The Stroop and reverse-Stroop 
tests were administered in each classroom in the middle of December in 2007.

\section{Results and Discussion}

Table 1

Descriptive Statistics and Shapiro-Wilk Test

\begin{tabular}{|c|c|c|c|c|c|c|c|c|c|c|c|c|c|}
\hline \multirow[b]{2}{*}{ Sections } & \multirow{2}{*}{$\begin{array}{l}\text { Full } \\
\text { Marks }\end{array}$} & \multicolumn{4}{|c|}{ TOTAL $N=68$} & \multicolumn{4}{|c|}{ Upper $n=22$} & \multicolumn{4}{|c|}{ Lower $n=22$} \\
\hline & & $M$ & $S D$ & $M d n$ & $p$ & $M$ & $S D$ & $M d n$ & $p$ & $M$ & $S D$ & $M d n$ & \\
\hline $1: \mathrm{JT} 1$ & 100 & 50.93 & 12.72 & 50.00 & .002 & 58.82 & 13.66 & 57.50 & .001 & 43.86 & 10.74 & 46.50 & .472 \\
\hline :JT2 & 50 & 43.71 & 9.71 & 45.00 & .079 & 50.14 & 8.07 & 48.50 & & 8.68 & & & \\
\hline JT3 & & $\overline{39.54}$ & 6.65 & 40.00 & .263 & 44.05 & 5.2 & 44.00 & 287 & 36.27 & 7.05 & 35.50 & .712 \\
\hline $\mathrm{JT} 4$ & 100 & 36.46 & 8.16 & 37.50 & .030 & 40.36 & 6.14 & 40.00 & .939 & 32.55 & 10.23 & 33.50 & 525 \\
\hline 5:JRSTI & & 12.15 & 18.11 & 13.00 & .000 & 12.99 & 11.87 & 13.15 & .078 & 7.97 & 27.48 & 6.83 & .002 \\
\hline JST & & 8.12 & 14.18 & 8.61 & .000 & 8.26 & 9.55 & 8.20 & .997 & 11.98 & 18.16 & 11.07 & .001 \\
\hline ET1 & 100 & 36.90 & 15.62 & 39.50 & .025 & 44.55 & 12.58 & 45.50 & 011 & 27.73 & 15.72 & 25.50 & .195 \\
\hline $8:$ ET2 & 100 & 34.82 & 12.28 & 37.50 & .027 & 41.23 & 9.69 & 41.50 & 013 & 26.36 & 11.99 & 27.00 & 28 \\
\hline 9:ET3 & 100 & 32.18 & 9.88 & 33.00 & .001 & 37.09 & 7.67 & 39.00 & 011 & 26.14 & 9.78 & 27.00 & .033 \\
\hline 10:E & 100 & 29.71 & 9.84 & 30.50 & .244 & 35.55 & 7.99 & 37.00 & .440 & 24.32 & & 26.00 & \\
\hline $1: \mathrm{E}$ & & -2.33 & 36.91 & 4.50 & .000 & 3.93 & 19.15 & 4.50 & .000 & -8.04 & 55.15 & 10.32 & .000 \\
\hline $12: \mathrm{ES}$ & & .81 & 44.14 & 8.63 & .000 & 2.04 & 20.47 & 5.51 & .002 & -11.09 & 71.06 & 6.97 & .000 \\
\hline $13: W \& P$ & 26 & 23.03 & 2.24 & 24.00 & .000 & 25.09 & .75 & 25.00 & .000 & 20.68 & 2.06 & 20.50 & .390 \\
\hline $1: \mathrm{C}$ & 11 & 9.72 & 1.41 & 10.00 & .000 & 10.59 & .59 & 11.00 & .000 & 8.50 & 1.63 & 8.50 & 064 \\
\hline $\mathrm{S}$ & & 6.84 & .92 & 7.00 & .000 & 7.45 & .74 & 8.00 & .000 & 6.45 & .80 & 7.00 & $\overline{002}$ \\
\hline $5: \mathrm{BT}$ & 45 & 39.59 & 3.39 & 40.00 & .023 & $\overline{43.14}$ & 1.13 & 43.00 & 001 & 35.64 & 2.26 & 36.00 & .006 \\
\hline
\end{tabular}

Notes. JT1 $=$ task 1 of the Japanese version of test. Each of JT2-JT4 means tasks 2-4 of the Japanese version of test. JRSTI = Japanese version of reverse-Stroop test interference rate as a percentage. JSTI = Japanese version of Stroop test interference rate as a percentage. ET1 = task 1 of the English version of test. Each of ET2-ET4 means tasks 2-4 of the English version of test. ERSTI = English version of reverse-Stroop test interference rate as a percentage. ESTI $=$ English version of Stroop test interference rate as a percentage. $\mathrm{W} \& \mathrm{P}=$ section of words and phrases in Bronze. $\mathrm{C}=$ section of conversation in Bronze. $\mathrm{S}=$ section of sentences in Bronze. $\mathrm{BT}=$ total marks in Bronze. Formula to calculate reverse-Stroop interference rate $=($ task $1-$ task 2$) /$ task $1 \times 100$. Formula to calculate Stroop interference rate $=($ task $3-$ task 4$) /$ task $3 \times 100$.

As for the results of normal distribution by Shapiro-Wilk test, shown in Table 1, some sections in the Stroop tests as well as Bronze were different at a significant level $(p<.05)$. Consequently, I analyzed all of these data with non-parametric tests by SPSS Ver.17.0. The upper and lower groups each were classified into about one-third of the total number of students, following the data of total marks of Bronze.

\subsection{Comparisons of Attention Abilities (Tasks 1 to 4) and Selective Attention Abilities (Two Interference Rates) Between $\mathrm{L} 1$ and EFL}

As shown in Table 2, as for tasks 1 and 2 (attention abilities concerning written words) and tasks 3 and 4 (attention abilities concerning colors), effect sizes of all 4 tasks in total were medium, and those in both upper and lower groups of English proficiency were large or medium. These results showed significant higher attention abilities of L1 than those of EFL. Therefore, research question 1 has been proved. This can be considered to show that language processing abilities of L1 are more 
predominant than those of FL.

Table 2

Wilcoxon Signed-Rank Test in Both Japanese and English Versions of Stroop and Reverse-Stroop Tests

\begin{tabular}{|c|c|c|c|c|c|c|c|c|c|c|c|c|}
\hline \multirow{2}{*}{$\begin{array}{c}\text { Stroop } \\
\text { Sections }\end{array}$} & \multicolumn{4}{|c|}{ Total $(N=68)$} & \multicolumn{4}{|c|}{ Upper $(n=22)$} & \multicolumn{4}{|c|}{ Lower $(n=22)$} \\
\hline & $Z$ & $p$ & $r$ & & $Z$ & $p$ & $r$ & & $Z$ & $p$ & $r$ & \\
\hline 1:JT1 - ET1 & -4.947 & .000 & 43 & M & -2.955 & .003 & .45 & $\bar{M}$ & -3.507 & .000 & .53 & $\mathrm{~L}$ \\
\hline 2:JT2 - ET2 & -4.264 & .000 & 37 & $\bar{M}$ & -3.330 & .001 & .50 & $\mathrm{~L}$ & -3.557 & .000 & .54 & $\mathrm{~L}$ \\
\hline 3:JT3 - JT3 & -4.555 & .000 & 39 & M & -3.985 & .000 & .60 & $\mathbf{L}$ & -4.110 & .000 & .62 & $\mathrm{~L}$ \\
\hline 4:JT4 - ET4 & -4.181 & .000 & 36 & M & -3.326 & .001 & .50 & $\mathrm{~L}$ & -3.722 & .000 & .56 & $\mathrm{~L}$ \\
\hline 5:JRSTI - ERSTI & -2.938 & .003 & 25 & $\bar{S}$ & -1.581 & 114 & .24 & $S$ & -.633 & .527 & .10 & $\mathrm{~N}$ \\
\hline 6:JSTI - ESTI & -.004 & 997 & .00 & $N$ & -1.088 & .277 & .17 & & 6 & 548 & .09 & $\bar{N}$ \\
\hline
\end{tabular}

Notes. $r=$ effect size. $\mathrm{S}=$ Small. $\mathrm{M}=$ Medium. $\mathrm{L}=$ Large. $\mathrm{N}=$ None. $r=.01<$ small, $.03<$ medium, .05 $<$ large. $n=$ the number of participants in each group. Calculating the effect size, $N$ or $n$ has to be interpreted as the number of observations, not the number of participants. Therefore, we must use this double number to calculate the effect size, and $N$ comes to 136 people, and $n 44$ people, that is, the number of participants (22) times 2.

On the contrary, as for two kinds of interference rates, effect size of reverse-Stroop interference in the total was small and that of Stroop interference in the total was not found to be significant. Effect sizes of the interference rates in the upper group were small and none were found in the lower one. Therefore, the results of two interference rates did not show remarkable differences of selective attention abilities between two languages. Therefore, research question 2 has not been proved. This could show that automaticity is not developed much in either L1 or EFL language processing. However, as for the mean of two interference rates, the scores of the Japanese version in the total, the upper group and the lower group turned out higher than those of the English version (Table 1). Regarding the median of two interference rates in the upper group, that of reverse-Stroop interference rate in the total, and that of Stroop interference rate in the lower group, the Japanese version was higher than the English one (Table 1), though the discussion of this point needs further consideration. In any case, this might show that abilities of automaticity in $\mathrm{Ll}$ processing are more predominant than those of EFL processing in the total, the upper and the lower groups.

\subsection{Comparisons of Attention Abilities (Tasks 1-4) and Selective Attention} Abilities (Two Interference Rates) Between Upper Group and Lower Group of English Proficiency

As shown in Table 3, as for age, there was no effect size between the two groups of English proficiency. Therefore, it can be said that there is no difference concerning age, and no effect influenced by cognitive development. Besides, needless to say, Bronze scores of each section in the upper group were much higher than those in the 
lower groups, as effect sizes of each section were all large.

Table 3

Mann-Whitney Test of Stroop and Reverse-Stroop Tests Between Upper Group and Lower Group of English Proficiency

\begin{tabular}{|c|c|c|c|c|c|c|c|c|c|c|c|c|c|c|}
\hline \multirow{2}{*}{\begin{tabular}{|c} 
Japanese \\
Version
\end{tabular}} & \multicolumn{4}{|c|}{$\mathrm{U}(n=22)-\mathrm{L}(n=22)$} & \multirow{2}{*}{$\begin{array}{l}\text { English } \\
\text { Version }\end{array}$} & \multicolumn{4}{|c|}{$\mathrm{U}(n=22)-\mathrm{L}(n=22)$} & \multirow{2}{*}{$\begin{array}{r}\text { Bronze } \\
\text { \&Age }\end{array}$} & \multicolumn{4}{|c|}{$\mathrm{U}(n=22)-\mathrm{L}(n=22)$} \\
\hline & $Z$ & $p$ & $r$ & & & $Z$ & $p$ & $r$ & & & $Z$ & $p$ & $r$ & \\
\hline $1: \mathrm{JT1}$ & -3.61 & .000 & .54 & $\mathrm{~L}$ & 7:ET1 & -3.39 & .001 & .51 & $\mathrm{~L}$ & 13:W\&P & -5.47 & .000 & .83 & $\mathrm{~L}$ \\
\hline 2:JT2 & -3.76 & .000 & .57 & $\mathrm{~L}$ & 8:ET2 & -3.81 & .000 & .57 & $\mathrm{~L}$ & $14: \mathrm{C}$ & -4.48 & .000 & .68 & $\mathrm{~L}$ \\
\hline 3:JT3 & -3.52 & .000 & .53 & $\mathrm{~L}$ & 9:ET3 & -3.95 & .000 & .60 & $\mathrm{~L}$ & 15:S & -3.71 & .000 & .56 & $\mathrm{~L}$ \\
\hline 4:JT4 & -2.69 & .007 & .41 & $\bar{M}$ & 10:ET4 & -3.67 & .000 & .55 & $\mathrm{~L}$ & $16: \mathrm{BT}$ & -5.73 & .000 & .86 & $\mathrm{~L}$ \\
\hline 5:JRSTI & -0.92 & 360 & .14 & $\mathrm{~S}$ & 11:ERST & -0.20 & .842 & -.03 & $\mathrm{~N}$ & 17:Age & -.32 & .752 & .05 & $\mathrm{~N}$ \\
\hline 6:JSTI & -0.56 & .573 & -.09 & $\mathrm{~N}$ & 12:ESTI & -0.56 & .573 & .09 & $\bar{N}$ & & & & & \\
\hline
\end{tabular}

Notes. $\mathrm{U}=$ Upper group in the Bronze test. $\mathrm{L}=$ Lower group in the Bronze test. $r=$ effect size. $\mathrm{S}=$ Small. $M=$ Medium. $L=$ Large. $N=$ None. Negative effect size means that the score in the upper group is higher than that in the lower group.

Concerning four tasks in Japanese version, effect sizes of tasks 1,2 and 3 were large, and that of task 4 was medium. As for two kinds of interference rates, effect size of reverse-Stroop interference rate was small, while no effect size of the Stroop interference rate was found. Concerning four tasks in the English version, effect sizes of all four tasks were large. On the other hand, effect sizes of reverse-Stroop and Stroop interference rates were not found. Overall, in Japanese and English versions, the number of correct responses in all four tasks in the upper group was considerably higher than those in the lower one, while differences in the two interference rates of both versions were not found. Therefore, research question 3 has been proved, but research question 4 has not been proved.

Concerning attention abilities, the participants in the upper group have higher attention abilities in both Japanese and English words. Therefore, the results can indicate that the upper group of English proficiency has higher processing abilities of language itself, including $\mathrm{L} 1$ and EFL.

As for selective attention abilities of both Japanese and English, though there was no significant difference between the upper group and the lower one, the features based on Table 1 are considered as follows. Regarding the Japanese version of interference rates, Stroop effect of the upper group was higher than that of the lower group, and reverse-Stroop effect was vice versa (the lower group was higher than the higher group). On the contrary, as for interference rates in the English version, both Stroop and reverse-Stroop effects of the upper group are higher than those of the lower group. Both effects of the upper group are positive interference rates, while those of the lower group are negative interference rates. This might show that the lower group cannot process and store English automatically. However, as all the participants of this 
research are only at a beginner's level in English, future study is needed under the condition of remarkably different levels of English proficiency.

\subsection{Relation Between Bronze and Two Tests (Stroop and Reverse-Stroop)}

Table 4

Spearman Correlation Between Two Tests (Stroop and Reverse-Stroop) and Bronze-TOTAL $(N=68)$

\begin{tabular}{|c|c|c|c|c|c|c|c|c|c|c|c|c|c|c|c|}
\hline & 1 & 2 & 3 & 4 & 5 & 6 & 7 & 8 & 9 & 10 & 11 & 12 & 13 & 14 & 15 \\
\hline 1:JT1 & - & $.698^{* *}$ & $.636^{* *}$ & $.466^{* *}$ & $.456 *$ & .041 & $.293^{*}$ & $.461^{* *}$ & $.428^{* *}$ & $.386^{* *}$ & .049 & .234 & $.467^{* *}$ & $.348^{* *}$ & .206 \\
\hline $2: J T 2$ & & .. & $.697^{* *}$ & $.549^{* *}$ & $.253^{*}$ & .083 & $.295^{*}$ & $.471^{* *}$ & $.487^{* *}$ & $.420^{* *}$ & .064 & $.251^{*}$ & $.528^{* *}$ & $.321^{* *}$ & .136 \\
\hline $3: J T 3$ & & & ... & $.802^{* *}$ & .053 & .132 & $.548 * *$ & $.636^{* *}$ & $.701^{* *}$ & $.705^{* *}$ & .121 & .165 & $.465^{* *}$ & $.356^{* *}$ & $.291^{*}$ \\
\hline 4:JT4 & & & & $\ldots$ & -.054 & $.681^{* *}$ & $.643^{* *}$ & $.670^{* *}$ & $.714^{* *}$ & $.781^{* *}$ & .150 & .160 & $.497^{* *}$ & $.405 * *$ & .194 \\
\hline $5: R S T I$ & & & & & $\ldots$ & .004 & .066 & .076 & .028 & .027 & .011 & .021 & .070 & .110 & .058 \\
\hline 6.JSTI & & & & & & .. & $.388^{* *}$ & $-360^{* *}$ & $.307^{*}$ & $.408^{* *}$ & .052 & -.071 & $.301^{*}$ & $-300^{*}$ & .048 \\
\hline 7:ET1 & & & & & & & $\ldots$ & $.892^{* *}$ & $.815^{* *}$ & $.756^{* *}$ & $.516^{* *}$ & $.269^{*}$ & $.522^{* *}$ & $.271^{*}$ & .234 \\
\hline 8:ET2 & & & & & & & & $\ldots$ & $.848^{* *}$ & $.739 * *$ & .198 & $378^{* *}$ & $.561^{* *}$ & $.426^{* *}$ & .233 \\
\hline 9:ET3 & & & & & & & & & $\ldots$ & $.864^{* *}$ & $.268^{*}$ & $.515^{* *}$ & $.473^{* *}$ & $387^{* *}$ & $.265^{*}$ \\
\hline 10:ET4 & & & & & & & & & & $\ldots$ & $.240^{*}$ & .171 & $.452^{* *}$ & $.308^{*}$ & $.362^{* *}$ \\
\hline 11:ERSTI & & & & & & & & & & & ... & .090 & .183 & $-.276^{*}$ & .191 \\
\hline 12:ESTI & & & & & & & & & & & & -- & .236 & $.323^{* *}$ & . 153 \\
\hline 13:W\&P & & & & & & & & & & & & & ..- & $.479^{* *}$ & .154 \\
\hline $14: C$ & & & & & & & & & & & & & & -- & .012 \\
\hline $15: S$ & & & & & & & & & & & & & & & - \\
\hline
\end{tabular}

Note. ${ }^{*} p<.05 . \quad{ }^{* *} p<.01$.

Table 5

Spearman Correlation Between Two Tests (Stroop and Reverse-Stroop) and Bronze-Upper Group of English Proficiency $\quad(n=22)$

\begin{tabular}{|c|c|c|c|c|c|c|c|c|c|c|c|c|c|c|c|}
\hline & 1 & 2 & 3 & 4 & 5 & 6 & 7 & 8 & 9 & 10 & 11 & 12 & 13 & 14 & 15 \\
\hline 1:JT1 & $\ldots$ & $.754^{* * *}$ & $.650^{* * *}$ & .360 & $.482^{*}$ & .150 & -215 & .152 & .132 & .060 & $.516^{*}$ & -044 & -.015 & .298 & .173 \\
\hline 2:JT2 & & -- & $.800^{* * *}$ & $.604^{* *}$ & -137 & .023 & -.005 & .394 & $.486^{*}$ & .288 & -333 & .075 & .263 & -.224 & .185 \\
\hline 3:JT3 & & & -. & $.740^{* * *}$ & * -.047 & .022 & .154 & $.427^{*}$ & $.581^{* *}$ & $.523^{*}$ & -175 & -178 & .365 & .009 & .132 \\
\hline 4:JT4 & & & & $\ldots$ & -228 & $-.594^{* *}$ & .221 & .262 & $.684^{* * *}$ & $.710^{* * *}$ & .078 & -.237 & $.458^{*}$ & .072 & .014 \\
\hline 5:RSTI & & & & & -. & .168 & -.173 & .189 & -.323 & -182 & -.260 & -.261 &. .118 & -.081 & .009 \\
\hline 6:JSTI & & & & & & -. & -.346 & -.072 & .361 & -.415 & -.400 & .059 & -.366 & .010 & .010 \\
\hline 7:ETl & & & & & & & -- & $.720^{* * *}$ & $.604^{* *}$ & $.467^{*}$ & $.652^{* * *}$ & -013 & $.553^{* *}$ & .298 & .063 \\
\hline 8:ET2 & & & & & & & & --- & $.724^{* * *}$ & .357 & .071 & .185 & $.549^{* *}$ & .114 & .325 \\
\hline 9:ET3 & & & & & & & & & $\ldots$ & $.699^{* \neq *}$ & .167 & .081 & $.697^{* * *}$ & .270 & .181 \\
\hline 10:ET4 & & & & & & & & & & -.- & .260 & $-.508^{*}$ & $.555^{* *}$ & .160 & .123 \\
\hline 11:ERSTI & & & & & & & & & & & -- & .007 & .214 & .289 & -.223 \\
\hline 12:ESTI & & & & & & & & & & & & $\ldots$ & .118 & -.061 & .135 \\
\hline 13:W\&P & & & & & & & & & & & & & -- & .169 & .085 \\
\hline $14: C$ & & & & & & & & & & & & & & - & -299 \\
\hline $15: S$ & & & & & & & & & & & & & & & $\ldots$ \\
\hline
\end{tabular}

Note. $\quad{ }^{*} p<.05 . \quad{ }^{* *} p<.01 . \quad{ }^{* * *} p<.001$ 
Table 6

Spearman Correlation Between Two Tests (Stroop and Reverse-Stroop) and Bronze-Lower Group of English Proficiency $\quad(n=22)$

\begin{tabular}{|c|c|c|c|c|c|c|c|c|c|c|c|c|c|c|c|}
\hline & 1 & 2 & 3 & 4 & 5 & 6 & 7 & 8 & 9 & 10 & 11 & 12 & 13 & 14 & 15 \\
\hline l:JTl & $\ldots$ & .342 & $.460^{*}$ & $.460^{*}$ & $.558^{* *}$ & -231 & .396 & $.490^{*}$ & $.546^{* *}$ & $.490^{*}$ & \begin{tabular}{l|l|}
.091 \\
\end{tabular} & .023 & .344 & .360 & .375 \\
\hline 2:JT2 & & -.- & $.663^{* * *}$ & $.513^{*}$ & -.386 & -114 & .221 & .390 & .320 & .255 & -.407 & .181 & .412 & .403 & $.509^{*}$ \\
\hline $3: J T 3$ & & & -- & $.914^{* * *}$ & -135 &. $.482^{*}$ & $.552^{* *}$ & $.642^{* * *}$ & $.646^{* * *}$ & $.667^{* * *}$ & .093 & .068 & .187 & .376 & -.088 \\
\hline 4:JT4 & & & & $\ldots$ & .097 & $-.778^{* * *}$ & $.671^{* * *}$ & $.722^{* * *}$ & $.711^{* * *}$ & $.764^{* * *}$ & .017 & -.119 & .285 & .411 & -.097 \\
\hline 5:RSTI & & & & & ... & .107 & .023 & -.007 & .124 & .111 & .076 & -.131 & .040 & .004 & .154 \\
\hline 6.JSTI & & & & & & .. &. $.586^{* *}$ & $.570^{* *}$ & $.501^{*}$ & $.621^{* *}$ & $\cdot 106$ & .217 & -.273 & -301 & .077 \\
\hline 7:ETl & & & & & & & $\cdots$ & $.903^{* * *}$ & $.899^{* * *}$ & $.818^{* * *}$ & .333 & .049 & .388 & .124 & .120 \\
\hline 8:ET2 & & & & & & & & -- & $.936^{* * *}$ & $.840^{* * *}$ & .007 & .112 & .358 & .357 & -.238 \\
\hline 9:ET3 & & & & & & & & & .. & $.902^{* * *}$ & .087 & .111 & .302 & .266 & -.141 \\
\hline 10:ET4 & & & & & & & & & & -- & .118 & -234 & .096 & .215 & .080 \\
\hline 11:ERSTI & & & & & & & & & & & ... & -.336 & .068 & $-.528^{*}$ & .373 \\
\hline 12:ESTI & & & & & & & & & & & & -. & .311 & .149 & $-.537^{* *}$ \\
\hline 13:W\&P & & & & & & & & & & & & & -.. & .078 & $.624^{* *}$ \\
\hline $14: C$ & & & & & & & & & & & & & & $\ldots$ & -.412 \\
\hline 15:S & & & & & & & & & & & & & & & $\ldots$ \\
\hline
\end{tabular}

Note. ${ }^{*} p<.05 . \quad{ }^{* *} p<.01 . \quad{ }^{* * *} p<.001$.

\subsubsection{Relation of Attention Abilities Between L1 and EFL}

As for correlation of tasks 1 to 4 between Japanese and English in the total, tasks 1 and 2 (attention to written words) of the reverse-Stroop test were positively correlated (task 1: $r=.293, p<.05$, task $2: r=.471, p<.01$ ), and tasks 3 and 4 (attention to colors) of the Stroop test were considerably correlated (task $3: r=.701, p$ $<.01$, task 4: $r=.781, p<.01$ ), as shown in Table 4. Concerning correlations of these tasks in the upper group of English proficiency, tasks 1 and 2 of the reverse-Stroop test were not significantly correlated. On the contrary, as for tasks 3 and 4 of the Stroop test, task 3 showed significant positive correlation $(r=.581, p<.01)$ and task 4 was also considerably correlated $(r=.710, p<.001)$, as shown in Table 5. Regarding correlations of these tasks in the lower group of English proficiency, the features were similar to the upper group; tasks 1 and 2 test did not correlate positively, and tasks 3 and 4 showed considerable positive correlations (task $3: r=.646, p<.001$, task $4: r$ $=.764, p<.001$ ), as shown in Table 6 .

Tasks 1 and 2, which primarily paid attention to written words in Japanese or English, require the quick activation of the meaning of words (verbal code) and the selection of the congruent color patches (generating imagery code, that is, color code). If the information processing speed of two languages in these tasks is similar or the same, there may be some positive correlation between them. However, these similar results of no correlation in the upper and lower groups are considered to be attributable to differences between L1 and EFL in processing abilities, accessing verbal code and generating imagery code from LTM. 
On the other hand, tasks 3 and 4 primarily paid attention to colors and selection of the congruent name of colors (generating verbal code). In task 4 , even if participants cannot activate meaning of color-words quickly, they only have to pay attention to only colors of color-words at first, and then they can select the word corresponding to the color of the ink. Therefore, if they have strategies of neglecting the meaning of color-words in Japanese as well as English, they can easily select the corresponding words, even though they cannot process English automatically. These significant positive correlations of tasks 3 and 4 can be considered to be due to the recognition of common concepts between the two languages, that is, colors, in accessing LTM. The results indicate that there would be similar processing abilities, accessing imagery code and generating verbal code from LTM between L1 and EFL.

\subsubsection{Relation of Attention Abilities in Stroop and Reverse-Stroop Tests}

Regarding correlation between Japanese reverse-Stroop tasks 1 and 2 in the total, it was considerably positive $(r=.698, p<.01)$. As for Japanese Stroop tasks 3 and 4 in the total, it was also considerably positive $(r=.802, p<.01)$. Likewise, English reverse-Stroop tasks 1 and 2 in the total showed considerable positive correlation $(r$ $=.892, p<.01)$ and English Stroop tasks 3 and 4 in the total also showed considerable positive correlation $(r=.864, p<.01)$.

These correlations can be considered as resulting from the focus of attention, that is, the attention to words in tasks 1 and 2, and the attention to colors in tasks 3 and 4.

Concerning correlation between Japanese reverse-Stroop tasks 1 and 2 in the upper group of English proficiency, it was considerably positive $(r=.754, p<.001)$. As for Stroop tasks 3 and 4 in the same group, it was also considerably positive $(r=.740$, $p<.001)$. Likewise, Stroop tasks 3 and 4 in the lower group also showed considerable positive correlation $(r=.914, p<.001)$, though correlation between Japanese reverse-Stroop tasks 1 and 2 was not significant $(r=.342, p=.120)$.

Regarding two pairs of correlation, that is, correlation between English reverse-Stroop tasks 1 and 2, and correlation between English Stroop tasks 3 and 4, they were considerably correlated in both the upper group (each correlation as follows: $r=.720, p<.001, r=.699, p<.001$ ) and the lower one (each correlation as follows: $r$ $=.903, p<.001, r=.902, p<.001$ ).

These considerable positive correlations in the English version of these tasks in two English proficiency groups can be also considered to be caused by the same attention. Furthermore, the considerable positive correlations in the Japanese version of these tasks in the upper group, might be evidence of efficient language processing abilities which are common to Japanese and English.

\subsubsection{Relation Between English Attention Abilities and English Proficiency}

The main results for correlation between each Bronze section and each task of 
English reverse-Stroop as well as English Stroop are as follows. In the total number of participants, the two sections of Bronze (the section of words and phrases, and the section of conversation) showed significant positive correlation in each of the four tasks (words and phrases section; correlation with task 1: $r=.522, p<.01$; with task 2 : $r=.561, p<.01$; with task $3: r=.473, p<.01$; with task $4: r=.452, p<.01$, conversation section; correlation with task 1: $r=.271, p<.05$; with task $2: r=.426, p<.01$; with task 3: $r=387, p<.01$; with task $4: r=.308, p<.05)$. As for the section of sentences, correlation was found only in tasks 3 and 4 (with task $3: r=.265, p<.05$; with task $4: r$ $=.362, p<.01$ ). Overall, correlations in smaller units of information, such as the section of words and phrases, tend to be higher than those of larger and complex units of information, such as the two other sections in Bronze.

In the upper group, the section of Bronze which showed significant positive correlation was only the words and phrase section (correlation with task $1: r=.553, p$ $<.01$; with task 2: $r=.549, p<.01$; with task $3: r=.697, p<.001$; with task $4: r=.555, p$ $<.01)$. No correlation in the two other sections, conversation and sentences, was found. Judging from the results of only correlation between the words and phrases section and the four tasks, "words," i.e. smaller units of information, can be considered as the common factor in information processing, because units of "words" play a central role of information processing for the four tasks as well as for the words and phrases section in Bronze, whereas sentences occurring in sections of conversation and sentences carry a heavier information load, and these larger units of information impose heavier burdens on participants. The heavier burdens make the participants access and storage information ineffectively. Therefore, it might be considered that no correlation occurs.

In the lower group, no correlation in all the three sections of Bronze was found. Judging from the results here of no correlation between the words and phrases section and the four tasks, which are correlated in the upper group, the cause of this absence of correlation might be this group's lower abilities of English language processing and accessing appropriate information from LTM.

\subsubsection{Relation Between Selective Attention Abilities in L1 and EFL}

No correlation between two interference rates of the Japanese version was found in the total or the upper group or the lower one. Similarly, in the English version, no correlation was found in the total or the upper group or the lower one. As Hakoda and Sasaki (1990) discussed about the Japanese version, the object of selective attention in Stroop test is "colors" and that of selective attention in reverse-Stroop test is "written words," and there appear to be different kinds of task switching of attention in the English version as well as the Japanese version. Therefore, it might be considered that these differences are the cause of no correlation between these two interference rates. 


\subsubsection{Relation Between English Selective Attention Abilities and English Proficiency}

As for correlation between two English interference rates and each Bronze section, one negative correlation (correlation with reverse-Stroop interference rate and conversation section; $r=-.276, p<.05$ ) and one positive correlation (correlation with Stroop interference rate and conversation section; $r=.323, p<.01$ ) were found in the total number of participants. On the other hand, no correlation was found in the upper group, whereas, in the lower group, two negative correlations were found (correlation with reverse-Stroop interference rate and conversation section; $r=-.528, p<.05$, one with Stroop interference rate and sentences section; $r=-.537, p<.01$ ). The results could show that automaticity in English language processing is not developed so much, irrespective of English proficiency. These negative significant correlations could be considered as evidence that the lower group has poor English task switching of attention in more complicated language information.

\section{Conclusion}

This study has found the following main four points. The first is that there were remarkable results concerning attention abilities: (1) Language processing abilities of L1 are more predominant than those of FL. (2) The upper group of English proficiency has higher processing abilities of language itself, including L1 and EFL.

The second is that there were no remarkable differences in selective attention abilities, neither between L1 and EFL nor between higher and lower English proficiency: (1) Automaticity does not develop so much in not only L1 but also EFL language processing. (2) Abilities of automaticity in Ll processing might be more predominant than those of EFL processing in the total, the upper and the lower groups. (3) The lower group might not be able to process and store English automatically.

The third is that there were remarkable different positive correlations in attention abilities between L1 and EFL, irrespective of English proficiency: There were different processing abilities, accessing verbal code and generating imagery code from LTM between $\mathrm{L} 1$ and EFL, while, there were similar processing abilities, accessing imagery code and generating verbal code from LTM between L1 and EFL.

The fourth is that there were remarkable positive correlations between attention abilities and three sections of Bronze, while there was no correlation between selective attention abilities and those sections: (1) It might be that the upper group had greater processing abilities to attend to smaller units of information (as words and phrases). (2) It might be that the attention abilities of the lower group to task switching was poor in more complicated English language information, judging from negative significant correlations between selective attention abilities and two sections of Bronze (conversation, sentences). 
Judging from the above results of this research, I think that they provide a certain level of scientific evidence of the effects "English activities" have on attention abilities (processing abilities), selective attention abilities (automaticity) in access and storage of two languages ( $\mathrm{Ll}$ and EFL) for about three years, though vocabulary as stimuli are very limited from the micro level of point of view. As pedagogical implications for foreign language activities, the following could be proposed:

1. The ability to pay accurate attention to higher frequency words (such as five color words, etc.) in EFL as well as $\mathrm{Ll}$ is vital in the comprehension of English (especially words and phrases). It is important for teachers to help students pay attention to a variety of vocabulary repeatedly, accurately and quickly by using a variety of effective teaching materials.

2. Automaticity of $\mathrm{Ll}$ as well as EFL in elementary school higher grade students does not develop so much and is at an immature cognitive development stage. Therefore, it is difficult for the students to perform speedy and complex information processing in foreign language activities once a week. The setting up of an "English environment", with a variety of foreign language activities (with frequent English language input, such as notices in English, events conducted in English, for example), would be beneficial in the development of attention abilities, and thus to the development of greater automaticity in the future.

However, needless to say, there were some limits in this study, such as the small number of participants of poor English language proficiency. Besides, there was incongruity between the visual mode in Stroop and reverse-Stroop tests and the aural mode in Bronze. Even if they were able to read words of five colors in this research, it may have been much easier for them to hear the words being spoken. From the cross-sectional sequential point of view, further research is needed with a greater variety of participants (for example, concerning their age, English proficiency and learning experiences which are affected by teaching methods, etc.). Still, not only within-condition but also between-condition, bilingual Stroop or reverse-Stroop tests are further needed in the same modality.

\section{Notes}

1: In this paper, "English activities" and "foreign language activities" are used synonymously. The participants in this research were taught English in "English activities." The goals and curriculum of the school cooperating in this research were the almost same as those of "foreign language activities" that the Ministry of Education, Culture, Sports, Science and Technology introduces in the new course of study.

2: As for the interpretation of the Stroop effect, that is, selective attention ability or interference rate, there are two prevalent accounts, "relative speed of processing" and "automaticity" (MacLeod, 1991). In this paper, I interpret it as 
"automaticity."

3: In this paper, second language (L2) and foreign language (FL) are used synonymously.

4: Jidoeiken, Bronze is a listening comprehension test for elementary school children. Judging from the goals and curriculum for English activities, this test has considerable validity in the evaluation of the English proficiency of the participants in this research. Stroop and reverse-Stroop tests, however, have four tasks which process information through visual code. Evaluating English proficiency for elementary school students in visual code (alphabet) is not appropriate, and there has been as yet no reliable English test for these students that use visual code. Thus, I regarded Bronze as an appropriate index to evaluate English proficiency in this research, though there are different modalities between Bronze and Stroop and reverse-Stroop tests.

\section{Acknowledgements}

This study was supported by a Grant-in-Aid for Scientific Research (C) (18520421) and was based on a research presentation at the $8^{\text {th }}$ Tsukuba International Conference on Memory in 2010. I would like to thank those teachers and students who have cooperated with this research, and also Dr. Yuji Hakoda and Miss Aki Matsumoto for their valuable comments.

\section{References}

Brauer, M. (1998). Stroop Interference in Bilinguals: The Role of Similarity Between the Two Languages. In A. F. Healy \& L. E. Bourne, Jr. (eds.), Foreign Language Learning: Psycholinguistic Studies on Training and Retention. Mahwah: Lawrence Erlbaum Associates. 317-337.

Butler-Goto, Y., \& Takeuchi, A. (2006a). Shogakko eigo katsudo ni okeru hyoka:Jidoeiken (BRONZE) wo tsukatta kokoromi [Evaluation of English activities at Japanese elementary schools: An examination based on the Junior STEP BRONZE Test]. JASTEC, 25, 1-15.

Butler-Goto, Y., \& Takeuchi, A. (2006b). Shogakko eigo katsudo ni okeru shido to communication noryoku: Jidoeiken Silver ni yoru chosa[Instruction and communication abilities:An examination based on the Junior STEP Silver Test]. STEP BULLETIN, 18, 248-263.

Chen, H. C. \& Ho, C. (1986). Development of Stroop interference in Chinese-English bilinguals. Journal of Experimental Psychology: Leaning, Memory, and Cognition, 12, 397-401.

Flowers, J. H. (1975). "Sensory" interference in a word-color matching task. Perception \& Psychophysics, 18, 37-43.

Hakoda, Y. \& Sasaki, M. (1990). "Shudanyo Stroop / Gyaku Stroop Test- 
Hannoyoshiki, Junjyo, Renshukoka" [GROUP VERSION OF THE STROOP AND REVERSE-STROOP TEST-The effects of reaction mode, order and practice-] Japanese Journal of Educational Psychology, 38, 389-394.

Hakoda, Y. \& Sasaki, M. (1991). "Shin Stroop Kensa" ni okeru nishu no kansho to hanno yoshiki. [Two kinds of interference in group version of the Stroop and reverse-Stroop test: The effects of reaction mode] Counseling-gakka Ronshu [Reports of Counseling and Guidance for Students], 5, Kyushu University. 69-81.

Hakoda, Y., Hirai, Y., Shina, K., \& Yanai, H. (2002). "Gakugyo seiseki to ninchi noryoku no kankei ni tsuite:Chui noryoku, gakuryoku shiken, ronjutsushiki kadai no sogo kankei wo chushin toshite" Research Project, Grant-in-Aid for Scientific Research on Priority (B) (1999-2001) "Daigaku Nyugakusha Senbatsu Shiryo toshiteno Sogo Shiken no Kaihatsuteki Kenkyu" Kenkyu Seika Hokokusho. 57-68.

Hakoda, Y. \& Watanabe, M. (2005). The Original Japanese Version of Stroop and Reverse-Stroop Test II. Fukuoka: Toyo Physical.

Hakoda, Y., Watanabe, M., \& Matsumoto, A. (2007). The English Version of Stroop and Reverse-Stroop Test II. Fukuoka: Toyo Physical.

Ikeda, S., Matsumi, N., \& Mori, T. (1994). "Eigo-Nihongo kan de shojiru gengonai/gengokan Stroop koka no kento: daigakusei to chugakusei no hikaku." [Stroop and Reverse Stroop Interference in English and Japanese] The Japanese Journal of Developmental Psychology, 5(1), 31-40.

MacLeod, C. M. (1991). Half a Century of Research on the Stroop Effect: An Integrative Review. Psychological Bulletin, 109(2), 163-203.

Mägiste, E. (1984). Stroop tasks and dichotic translation: The development of interference patterns in bilinguals. Journal of Experimental Psychology: Learning, Memory, and Cognition, 10, 304-315.

Osaka, M. (1990). "Bairingaru to Stroop koka" [Bilingualism and Stroop effect] Journal of Osaka University of Foreign Studies, 4, 77-87.

Posner, M. I. \& Snyder, C. R. R. (1975). Facilitation and inhabitation in the processing of signals. In P. M. A. Rabbitt \& S. Dornic (Eds.) Attention and performance, 15. 669-682. New York: Academic Press.

Pritchatt, D. (1968). An investigation into some of the underlying associative verbal processes of the Stroop colour effect. Quarterly Journal of Experimental Psychology, 20, 351-359.

Robinson, P. (2003). Attention and Memory during SLA. In C. Doughty \& M. Long (Eds.), Handbook of Second Language Acquisition. 631-678. Malden, MA: Blackwell.

Sakuma, Y. (2009). Changes in Listening Ability and Psychological Factors Influenced by Elementary School English Activities. Annual Review of English Language Education in Japan, 20, 221-230. 
Sakuma, Y. (2010). Gaikokugokatsudo Ikoki ni okeru Koritsu Shogakko Chukaku Kyoin no Ishikichosa: Genjyo to Kadai. [Research on Consciousness of Chief Teachers at Public Elementary Schools during Foreign-Language Activities Transitional Period: the Present Condition and Issues] Tohoku Eigo Kyoiku Gakkai Kenkyu Kiyo 30, 29-43.

Stroop, J. R. (1935). Studies of interference in serial verbal reactions. Journal of Experimental Psychology, 18, 643-662.

Takada, T. (2008). A Longitudinal Study of the Effects of Learning English in Elementary School. Annual Review of English Language Education in Japan, 19, 231-240.

\section{Appendix}

Practice Section of The English Version of Stroop and Reverse-Stroop Test II Practice

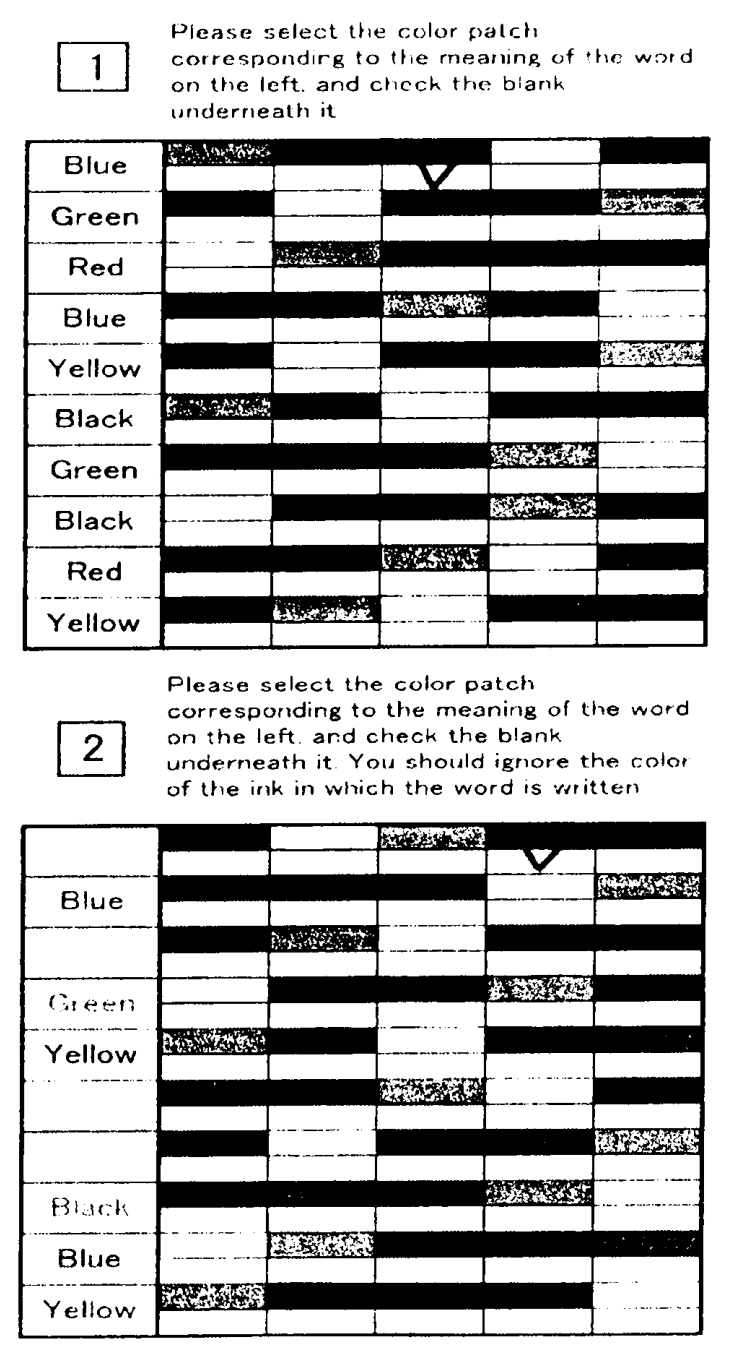

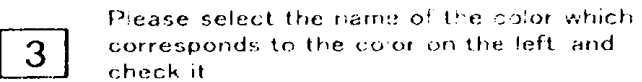

\begin{tabular}{|c|c|c|c|c|c|}
\hline & Yelbw & Blue & Green & Black & Red \\
\hline Blue & Yellow & Black & Red & Green \\
\hline Black & Red & Blue & Green & Yellow \\
\hline Green & Black & Red & Elue & Yellow \\
\hline Red & Green & Yellow & Black & Blue \\
\hline & Blue & Red & Green & Yellow & Black \\
\hline & Black & Yellow & Blue & Red & Green \\
\hline Green & Black & Red & Yellow & Blue \\
\hline & Red & Blue & Yellow & Green & Black \\
\hline Yellow & Green & Black & Blue & Red \\
\hline
\end{tabular}

From the color words, wr tten in black. please select the word corresponding to

4 the color of the ink used to write the

color words on the far eft and check it You stould ignore the meaning of each

\begin{tabular}{|c|c|c|c|c|c|}
\hline Red & Green & Black & Red & Blue & Yellow \\
\hline Green & Black & Yellow & Blue & Green & Red \\
\hline & Blue & Green & Yellow & Red & Black \\
\hline & Yellow & Red & Blue & Black & Green \\
\hline Black & Green & Blue & Yellow & Black & Red \\
\hline Green & Yellow & Blue & Black & Red & Green \\
\hline & Blued & Black & Red & Green & Yellow \\
\hdashline & Red & Green & Yellow & Blue \\
\hline Red & Yellow & Green & Blue & Black \\
\hline
\end{tabular}

